\title{
Thoracoscopic repair of Sibson hernia in a pediatric patient
}

\author{
Ruchi Amin, MD, ${ }^{a}$ Veronica F. Sullins, MD, ${ }^{a}$ Michael E. Mitchell, MD, ${ }^{b}$ and Marjorie J. Arca, MD, \\ Milwaukee, Wis
}

\author{
From the Divisions of ${ }^{\mathrm{a} P e d i a t r i c}$ General and Thoracic Surgery and ${ }^{\mathrm{b}}$ Pediatric Cardiothoracic Surgery, Medical \\ College of Wisconsin, Children's Hospital of Wisconsin, Milwaukee, Wis. \\ This research was not supported by grants or funding agencies in the public, commercial, or not-for-profit sectors. \\ Disclosures: Authors have nothing to disclose with regard to commercial support. \\ Received for publication June 21, 2018; revisions received July 25, 2018; accepted for publication July 28, 2018; \\ available ahead of print Oct 22, 2018. \\ Address for reprints: Marjorie J. Arca, MD, 999 N 92nd St, Pediatric Surgery, Unit 320, Milwaukee, WI 53226 \\ (E-mail: marca@chw.org). \\ J Thorac Cardiovasc Surg 2019;157:e187-8 \\ $0022-5223 / \$ 36.00$ \\ Copyright (C) 2018 by The American Association for Thoracic Surgery \\ https://doi.org/10.1016/j.jtcvs.2018.07.110
}

Video clip is available online.

\section{CLINICAL SUMMARY}

A 13-year-old girl was seen in clinic for exertional dyspnea, lightheadedness, syncope, and right neck bulge. A $4-\mathrm{cm}$ defect with right lung herniation was seen on computed tomographic scan (Figure 1). Her symptoms were attributed to extrinsic compression of the right internal jugular vein from the hernia.

With the patient in the left lateral decubitus position, single-lung ventilation was accomplished. The normalappearing lung apex was reduced, revealing a $4-\mathrm{cm}$ defect posterior to the innominate vein and anterior to an aberrant right subclavian artery (Video 1). Excess hernia sac was excised, and the parietal pleura was closed taut with a running suture. We used a 1-mm polytetrafluoroethylene patch, allowing a 2-cm overhang of circumferential coverage beyond the fascial defect. The patch was secured with sutures and endoscrews. There was no visible herniation once the lung was reinflated. A chest radiograph after completion of the procedure appeared normal.

The patient was discharged on postoperative day 2. She had complete resolution of her symptoms at 4 weeks, with no evidence of recurrence at 1 year.

\section{DISCUSSION}

Cervical lung hernias through a defect in Sibson fascia are extremely rare in children and are often secondary to local external trauma or to thoracic surgical procedures. ${ }^{1}$ The Sibson fascia extends from the transverse process of C7 to insert on the inner border of the entire first rib and on the trachea. ${ }^{4}$

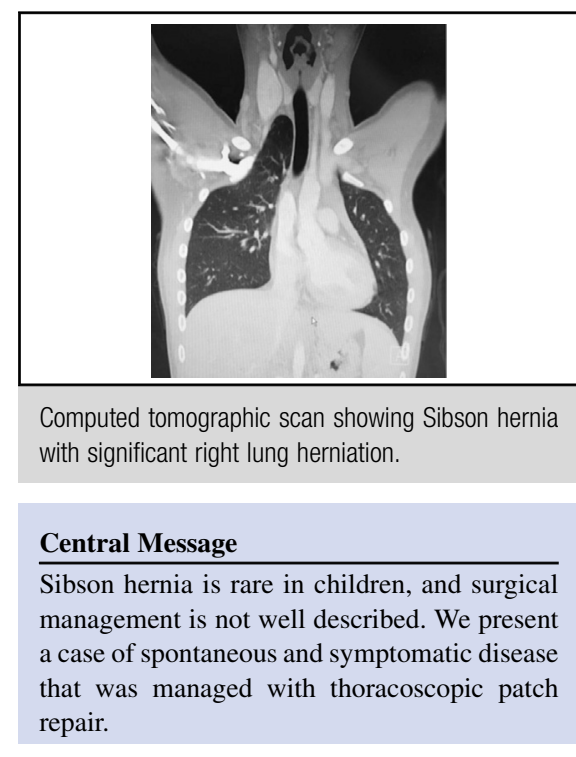

See Commentary on page e189.

borders medially on the superior mediastinal structures. This double layer of opposed fascia prevents ascent of the lung beyond the thoracic inlet. ${ }^{2}$

Sibson hernias occur when there is a partial absence, laxity, or attenuation of the fascia. Patients commonly present with a soft neck bulge that increases in size with a Valsalva maneuver. Although most patients do not have symptoms, some may report dysphagia, chronic cough, or even neuropathic pain. ${ }^{3}$ Our patient presented with shortness of breath and syncope exacerbated by activity as well as by playing wind instruments. The relationship of the hernia to the surrounding anatomy is best seen on computed tomography of the chest; in our case, the herniated lung could be seen to compress the jugular vein. Imaging modalities may help discriminate a true Sibson hernia from other neck pathology. A contrast esophagogram would rule out an esophageal diverticulum. Ultrasonography and axial imaging would determine other causes of neck swelling, such as lymphadenopathy, clavicular or first rib masses, or aneurysmal dilation of the subclavian artery. To distinguish simple lung hernia from a true Sibson hernia, lateral neck films with the patient performing a Valsalva maneuver are helpful. ${ }^{4}$ Fluoroscopy in frontal and oblique projections during straining may show the effect of lung protrusion 


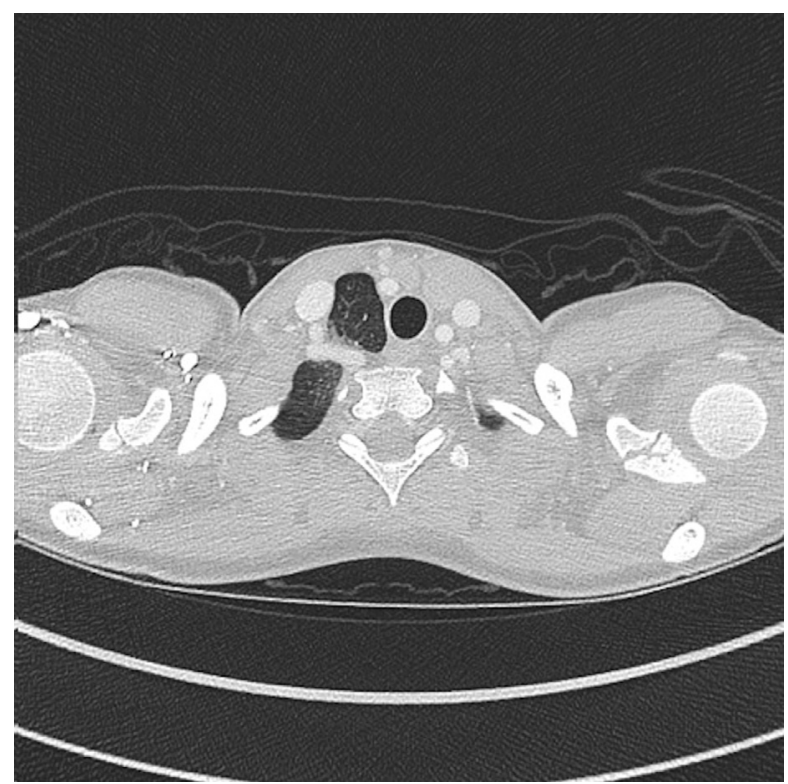

FIGURE 1. Computed tomographic scan of the chest (transversely at the level of the cervical hernia) showing a 4-cm defect with significant right lung herniation in association with an aberrant right subclavian artery.

Surgical repair of these hernias is uncommon, because the vast majority are asymptomatic, and the risk of incarceration is low. Simple lung protrusion in neonates often resolves by the age of 3 years and does not require intervention. ${ }^{4}$ For patients with true cervical hernia and clinically significant disease, however, surgical repair may be considered. Repair can be performed either in an open or thoracoscopic manner, and the defect can be repaired primarily, with tissue such as periosteum or muscle, or with synthetic material, according to the size of the defect. The first thoracoscopic repair reported in an adult also required resection of the lung as a result of emphysematous changes from air trapping. ${ }^{5}$ Our patient had a normal apical segment, and resection was not necessary. Jheon and colleagues ${ }^{5}$ described the use of a hernia stapler and endoscrews to fix the hernia sac to the chest wall to patch the defect. Ultimately, they found this to be inadequate and used intercostal muscle to reinforce their repair. In our case, we excised the redundant portion of the sac, closing the parietal pleura at the neck of the hernia. Our experience with biologic patches in diaphragmatic hernias demonstrated acquired redundancy with time. Polytetrafluoroethylene was therefore used to reinforce the repair where the Sibson fascia was missing. We secured the patch to the parietal pleura with interrupted nonabsorbable sutures in places that were

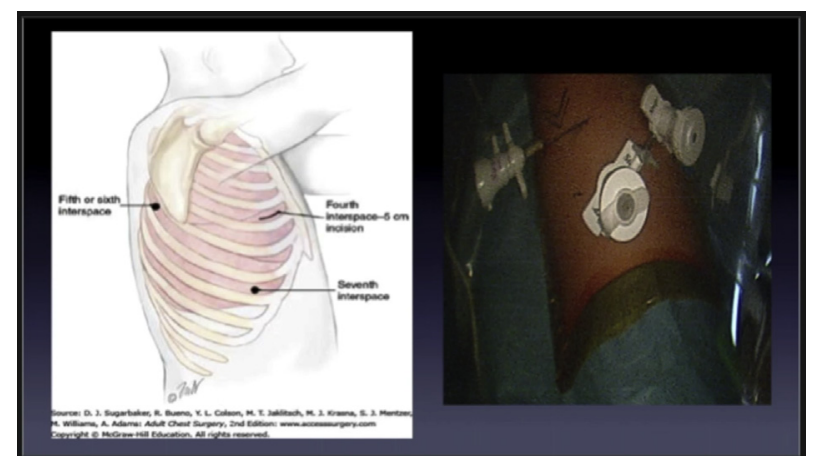

VIDEO 1. The patient was placed in the left lateral decubitus position. Preoperative bronchoscopy was performed with double-lumen endotracheal tube placement and isolation of the right lung. A 5-mm port was placed below the tip of the scapula posteriorly, a second $5 \mathrm{~mm}$ port was placed in the fifth interspace at the anterior axillary line, and finally a $10 \mathrm{~mm}$ port was placed in the seventh interspace at the midaxillary line. On thoracoscopic entry of the chest, the hernia was clearly visible, with no adhesions or attachments. The defect measured $4 \mathrm{~cm}$ and was located posterior to the innominate vein and anterior to the aberrant right subclavian artery. After partially incising the hernia sac, we carefully worked through the incision to identify and preserve the vagus and phrenic nerves and the internal jugular vein. After excision of the remaining hernia sac, the defect was closed with a running suture. An $8 \times 8$-cm circular patch of polytetrafluoroethylene was cut to size to allow an additional $2 \mathrm{~cm}$ of coverage beyond the fascial defect. Sutures were placed in all 4 quadrants before insertion into the thoracic cavity, with the adult technique for ventral hernia repair used. The patch was unfurled, and the sutures were secured to the parietal pleura. The patch was secured with additional sutures and endoscrews to ensure adequate apposition. A $20 \mathrm{~F}$ straight chest tube was left in the right pleural space, and the right lung was reinflated. Video available at: https://www.jtcvs.org/article/S0022-5223(18)32473-5/fulltext.

adjacent to neurovascular structures and endoscrews in areas away from critical structures.

This is a unique case of a spontaneous Sibson hernia in a pediatric patient with symptomatic disease. The patient was successfully treated with thoracoscopic reduction of the herniated lung, primary closure of the pleura, and patch reinforcement of the repair.

\section{References}

1. Weissberg D, Refaely Y. Hernia of the lung. Ann Thorac Surg. 2002;74:1963-6.

2. Miyake N, Takeuchi H, Cho BH, Murakami G, Fujiyama M, Kintano H. Fetal anatomy of the lower cervical and upper thoracic fasciae with special reference to the prevertebral fascial structures including the suprapleural membrane. Clin Anat. 2011;24:607-18.

3. Rahman M, Buchan KG, Mandana KM, Butchart EG. Bilateral cervical lung hernia with T1 nerve compression. Ann Thorac Surg. 2006;81:716-8.

4. Currarino G. Cervical lung protrusions in children. Pediatr Radiol. 1998;28:533-8.

5. Jheon S, Lee EB, Cho JY, Chang BH, Lee J, Kim KT. Thorascopic repair of cervical lung hernia. J Thorac Cardiovasc Surg. 2002;124:1030-1. 